\title{
A TEST OF RADIO INTERFEROMETRY BY LUNAR REFLECTION
}

\author{
L. BELKORA \\ Astrophysical, Planetary and Atmospheric Sciences Department, \\ University of Colorado, Boulder, CO 80309-0391
}

TOR HAGFORS AND J.A. PHILLIPS

N.A.I.C., Cornell University, Ithaca NY 14853

\begin{abstract}
We discuss the use of the moon as a passive reflector for radio interferometry experiments with baselines on the order of the radius of the orbit of the moon. Because of the extreme loss in the lunar interferometer path, only exceptionally strong point sources are candidates for this kind of interferometry. As the moon is far from an ideal reflector due to the irregular surface, the data processing necessary to optimize the fringe detectability is rather complicated. What we present here is a very brief summary of a paper describing the lunar interferometer in the Astrophysical Journal (Hagfors, Phillips and Belkora, 1990) and an experiment currently underway at Arecibo to test the lunar interferometer using Jupiter's strong bursts at $25 \mathrm{MHz}$ as a source. All references pertaining to the lunar interferometer are to be found in the Astrophysical Journal paper; this summary includes references concerning the test of the interferometer at Arecibo.
\end{abstract}

\section{INTRODUCTION}

The use of a passive reflector to produce interference fringes has been applied in various forms in radio observations for some time; the sea or "cliff" interferometer is an early example. The idea of lunar interferometry was discussed in the late 1960's at the MIT Lincoln Laboratory but the initial concept using $\mathrm{OH}$ maser emission from W3 as a source was abandoned because the expected signal-to-noise was too low. Artyukh and Shishov also outlined the moon interferometer scheme. Since then the idea has been revived by A.E.E. Rogers for possible studies of the very strong $\mathrm{H}_{2} \mathrm{O}$ emission from Orion. One of us (J.A. Phillips) has also proposed to use this technique to try to spatially resolve S-type Jupiter bursts at $25 \mathrm{MHz}$ which typically have a flux density of $10^{6}$ or $10^{7} \mathrm{Jy}$, and this experiment is currently underway. 


\section{DESCRIPTION OF THE EXPERIMENT}

Figure 1 shows the geometry of the lunar interferometer. At antenna A we receive a direct signal from the source (e.g. Jupiter). At antenna $B$ we record the signal as it is reflected from the moon. The effective baseline is shown as the line $\mathrm{D}$ in the figure. We call the angle subtended at the earth between the moon and source $\phi$, and with the Earth-moon distance as $R_{m}$ we have

$$
D \approx R_{m} \sin \phi-2 \mathrm{a} /(1+\cos \phi)
$$

where $\mathrm{a}$ is the radius of the moon.

The moon-reflected signal is highly attenuated and we seek grazingincidence geometry to minimize this effect. The condition of grazing incidence at the moon implies a small angle subtended at the earth between the moon and the source, i.e. small $\phi$. The relationship between the angle of incidence $\theta$ and the angular separation is $\theta \approx \frac{\pi}{2}-\frac{\phi}{2}$. Figure 2 shows the lunar reflectivity versus angle of incidence expressed as baseline in lunar orbital radii.

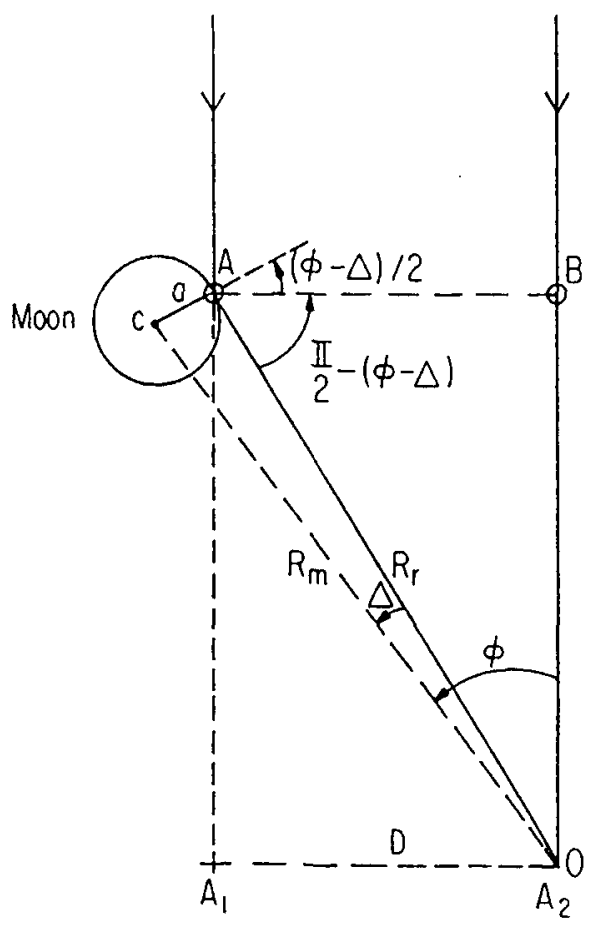

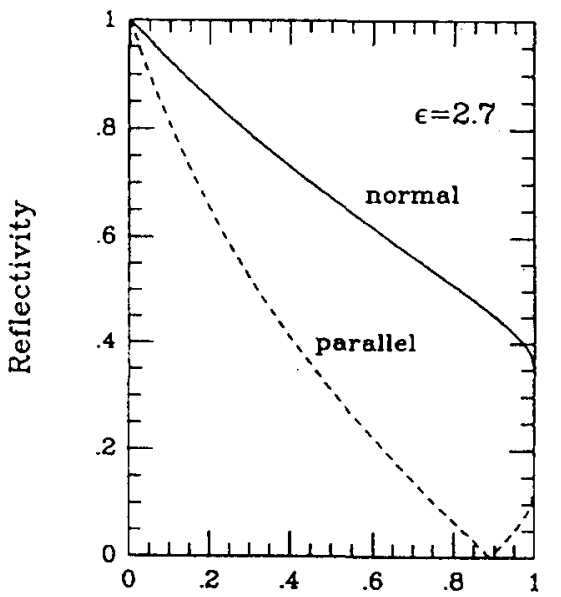

Baseline, units of orbital radi

Figure 1 (Left). Geometry of the lunar interferometer Figure 2 (Right). Lunar reflectivity versus baseline 
For $\phi$ between 1 and 2 degrees, the baseline is an Earth-diameter or about $10^{7} \mathrm{~m}$, and the resolution at $25 \mathrm{MHz}$ is about $0.2^{\prime \prime}$. For $\phi \sim 10^{\circ}$ the baseline is 5.1 Earth-diameters and the resolution at $25 \mathrm{MHz}$ is $0.04^{\prime \prime}$.

\section{A BRIEF DESCRIPTION OF THE JOVIAN DECAMETRIC SOURCES}

Decametric emission from Jupiter ("DAM") is organized on time scales of a few hours into storms, which peak in intensity whenever certain sub-Earth longitudes appear on Jupiter and when Io has the right orbital phase. How Io enhances the emission is not entirely understood; the viewing geometries which are best for detecting storms are, however, so well documented as to be predictable. We use a prediction ephemeris produced by the University of Florida, which gives hourly storm probabilities at 18 and $26 \mathrm{MHz}$.

DAM is interesting not only because of its high total power radiated during a typical storm, which may exceed $10^{9}$ Watts (Melrose, 1980), but also because it displays two distinct components, L- and S-bursts. L (for long) bursts last $\approx 1$ to 30 seconds, while the MegaJansky S (short) bursts last only hundredths of seconds and drift rapidly in frequency space.

Interferometry of Jupiter's DAM has been carried out for almost twenty years, and while some investigators may have resolved $L$ bursts with baselines of 50 to $200 \mathrm{~km}$ (Slee and Higgins, 1966), continental baselines and arc-minute resolution have not sufficed to resolve the S-bursts. (Dulk 1970, Lynch et al. $1972,1976)$. Using the lunar interferometer we hope to resolve the $S$ burst source with baselines considerably larger than the earth's diameter.

\section{PROCESSES AFFECTING THE MOON-REFLECTED SIGNAL}

\section{Surface Roughness.}

Instead of the return coming from a Fresnel zone centered on the geometric reflection point, the actual reflections arise from a large number of specular points associated with individual irregularities. The returns will be distributed over a sizeable fraction of the lunar surface and hence spread in time delay. This determines the bandwidth over which the phase of the visibility is constant. For example, if the delay range affecting the scattered signal is $13 \mu \mathrm{sec}$, as is the case when Jupiter is $10^{\circ}$ from the moon and the observing frequency is $25 \mathrm{MHz}$, the coherence bandwidth is $1 / 13 \mu \mathrm{sec}$ or about $80 \mathrm{kHz}$.

The roughness of the moon forces us to divide the reflected signal in delay range. To optimize the signal to noise we form the weighted average of visibility amplitudes in each delay interval. The weighting function is empirically determined from Earth-based radar experiments which give the reflected power as a function of lunar range.

\section{Rotations of the moon.}

The rotation and other motions of the moon Doppler broaden the reflected signal. The Doppler broadening determines the time for coherent integration: at $25 \mathrm{MHz}$ and for $\phi=10^{\circ}$ the Doppler width of the signal is $8 \mathrm{mHz}$ and the coherence time is thus 125 seconds. We are fortunate in this experiment in that the S-bursts have instantaneous bandwidths on the order of $10 \mathrm{kHz}$, 
much smaller than the $80 \mathrm{kHz}$ coherence bandwidth computed for the viewing geometry described above, and have a duration of about $100 \mathrm{msec}$, so that many bursts can be averaged coherently for a high signal to noise ratio.

The time dependence of the reflected signal which arises because different portions of the lunar surface rotate into the reflecting zone is discussed in the section of the paper entitled "The Rough Moon-Time Variation and Spread in Delay."

\section{CURRENT OBSERVATIONAL EFFORTS: THE JUPITER EXPERIMENT AT ARECIBO}

We have built a crossed Yagi antenna at Arecibo to detect the direct signal from Jupiter and installed it on a hilltop close to the dish. A $25 \mathrm{MHz}$ "feed" for the 1000 foot dish was constructed also: it consists of a set of crossed dipoles and a "quad" reflector. The next observing dates are November 10 and December 5, 1990, and in January and February 1991.

Previous attempts (in 1988 and 1989) have yielded only a few minutes of data, not enough for fringe detection; the most important obstacle is the scarcity of opportunity in the Jupiter-based version of the experiment. The moon must be visible to the dish at Arecibo (i.e. within $20^{\circ}$ of overhead) and Jupiter should be less than about twenty degrees from the moon, for grazing incidence; at the same time the phase of Io and the sub-Earth longitude on Jupiter must be such that an Io-related storm is predicted to occur at Jupiter. Furthermore, interference is such a problem at this low frequency that we can only attempt the experiment during winter nights. Only a few opportunities occur every winter.

\section{REFERENCES}

Calvert, W. 1982, J.G.R. 878199.

Carr, T. D., Desch, M. D. and Alexander, J. K. 1983,in

Physics of the Jovian Magnetosphere, ed. A. J. Dessler, Cambridge

University Press, New York, Chapter 7, 250.

Dulk, G. A. 1970, Ap. J. 159,671.

Genova, F. and W. Calvert. 1988, J. G. R. $93,979$.

Hagfors, T., Phillips, J. A., and L. Belkora. 1990, Ap. J. 362, 308.

Lynch, M. A., T. D Carr, J. May, W. F. Block, V. M. Robinson, and N. F. Six. 1972, Ap. J. 10, 153.

Lynch, M. A., T. D. Carr, and J. May. 1976, Ap. J. 207, 325.

Maeda, K., and T. D. Carr, in Proceedings of the Second Annual Workshop on Radio Emissions from Planetary Magnetospheres, Graz, Austria

Melrose, D. B. 1980, Plasma Astropysics: Nonthermal Processes in Diffuse Magnetized Plasmas. Volume II: Astropysical Applications (New York, N.Y.: Gordon and Breach Scientific Publishers.)

Slee, O. B. and C. S. Higgins. 1966, Aust. J. Phys. 19, 167. 